\title{
Self-organization as a fundamental factor in sustainable development and operation of continuing professional education
}

\author{
Elena Pesotskaya ${ }^{1, *}$, Larisa Selyutina ${ }^{2}$, Maria Ivanova $^{3}$, and Marina Voloshinova $^{1}$ \\ ${ }^{1}$ Saint-Petersburg State University of Economics, 21, Sadovaya str., 191023 St. Petersburg, Russia \\ ${ }^{2}$ Emperor Alexander I St. Petersburg State Transport University, 9, Moskovsky pr., 190031 St. \\ Petersburg, Russia \\ ${ }^{3}$ Saint Petersburg State University of Civil Aviation, 38, street of Pilots, 196210 St.Petersburg, Russia
}

\begin{abstract}
The article deals with the basic provisions of the theory of selforganization, characterizes their ability to modify the management process in various industries and economic spheres. The specificity of selforganization in relation to lifelong professional education, its sources, methodology, factors that need to be taken into account in the formation of managerial decisions are investigated. The article reflects the cyclical nature of continuing professional education, the content and structure of its inherent self-organization cycle with its ability to ensure sustainable development and functioning of the system of continuing professional education.
\end{abstract}

\section{Introduction}

Considering continuing professional education (CPE) as a system evaluated from the perspective of a number of theoretical and methodological approaches, it is worth emphasizing its capacity for self-organization resulting from the methodology determined by the fractal theory [1-3].

Fractals, fractal enterprises from the standpoint of the founders of fractal theory (H.-Y. Warneke, B. Mandelbrot, I.A. Prigozhin, G.Haken, etc.) are elements of the system capable of independent functioning and self-development, achieving the greatest effect through joint actions.

Self-organization is one of the key properties of a fractal. In modern studies, the phenomenon of self-organization, the connection between self-organization and organization as a function or process, is given considerable attention [4-7]. They note that the concept of "self-organization" arose in the course of the study of evolutionary processes and the formation of modern ideas about evolution as such.

One of the first steps in the formation of such ideas is the well-known scientific work of I.A. Prigogine [8], in which the following proposition is stated and substantiated: systems left to themselves, i.e. functioning on the principles of self-organization, can reduce their entropy (which contradicted the traditional scientific concepts of that period).

\footnotetext{
*Corresponding author: epes2@mail.ru
} 
The aim of the study is to study and analyze the phenomenon of self-organization and its scientific and methodological provisions, as well as the adaptation of these provisions to the specifics of continuing professional education, taking into account the cyclical nature of the self-organizing process.

\section{Materials and Methods}

The term "self-organization" was originally used to denote natural anti-entropic processes in nature, and then it gradually moved to the level of organizations and enterprises, fractal structures, social processes and society as a whole.

Basic for understanding the essence of self-organization is the differentiation of the concepts of "self-organization" and "organization". An unambiguous understanding on this issue was formulated by T.Yu. Ivanova, V.I. Prikhodko [9]. They noted that selforganization is a change in the state of an object in a natural way, and organization is a change in state that occurs artificially. Moreover, the prefix "self" demonstrates the immanent causes of this phenomenon, as well as the inherent possibility for establishing a certain order. Self-organization is presented as active ordering.

The theory of self-organization, which has expanded the boundaries of its application, operates with such terms as naturalness, integrity, universal interconnection, open systems, nonlinearity, disequilibrium, bifurcation, emergence, ambivalence.

A.I. Kochetkova noted the key properties of self-organization [10, p. 447-448], which fully correspond to the characteristics of continuing professional education. It emphasizes, first of all, the systemic nature of self-organization, the appearance of its signs in any conditions, dependence on resources (human and informational), as well as the presence of an element of randomness in self-organization.

As an important property of self-organization (as applied to CPE), one can single out its temporary nature and the possibility of having both reversible and irreversible character. Moreover, the irreversible character is linked both with the development of a selforganizing system and with its destruction. Destruction is considered in this case as a transition to another quality, to another state that preserves a minimum of stable properties of the old system (fractals).

In the case of the development of a self-organizing system, it is possible to minimize the risks, and when it is destroyed, this possibility disappears.

From the point of view of management, an important property of self-organization should be highlighted - it is implemented against the background of the formation of many short-term relationships (between groups of specialists, individual specialists or others belonging to the number of intersubjective), which, as a rule, disintegrate after solving a specific problem, but are capable to some extent persist when solving other problems [11]. In self-organization, management operates with virtual systems of quality indicators and parameters, the totality of which is due (in the case under consideration) to the specifics of the activities of organizations of continuing professional education.

Self-organization from the standpoint of management should be considered as a factor in increasing the uncertainty of the system. The use of hierarchical structures will help reduce uncertainty.

A.I. Kochetkova noted that self-organization effectively functions only in organizations with hierarchically structured structures - "the rigidity of the shell should be adequate to the power of the content - a chaotic (fractal) model of organizational behavior" [10, p. 448]. It is important to pay attention to the following circumstance: the hierarchy of self-organizing systems is combined with the possibility of disintegration of short-term connections that are formed to achieve individual tasks [12]. In educational organizations (CPE), this manifests 
itself in the form of maintaining a single hierarchical structure with changes in its individual divisions or groups of specialists.

The most comprehensive aspect of self-organization in continuing vocational education is the social aspect. Self-organization always relies on a person, on his desire and ability to participate in interactions between subjects and improve these interactions [13]. It requires a high level of development of a person involved in self-organization, mobilization of his professional qualities and creative abilities, laterality of thinking, communication qualities, striving for self-development, etc.

The instrumental support of self-organization is the use of monitoring tools [14]. In the CPE system, taking into account its self-organization, a wide variety of phenomena, processes, subsystems, organizational elements are formed, which are advisable to be included in the number of monitoring objects. Monitoring is intended to ensure observation of the parametric range of management of the CPE and its member organizations, as well as to ensure the implementation of such management functions as analysis and forecast.

In examining the phenomenon of self-organization, it is important to note the following methodological feature: there are two opposite ways of constructing structures - hierarchy and heterarchy. In the theory of organizations, it is noted that a person, as a subject of management, always strives to build hierarchical structures with their relations of subordination and centralization of power [15]. But a number of studies demonstrate the flaws of the hierarchy, which are manifested in the process of solving highly complex problems. Such tasks can be successfully solved only in structures devoid of authoritarianism and based on a polycentric distribution of powers, i.e. heterarchy. With heterarchy, hierarchical relationships are created only as necessary, to solve specific problems that require volitional efforts and special knowledge.

Moreover, as emphasized in [9], various combinations of hierarchical and heterarchical structures are possible. For example, in the system of continuing professional education, current activities can be organized on the principles of hierarchy and in a centralized environment. However, when carrying out large-scale reforms, it will be necessary to solve problems of an innovative type, in the implementation of which expert groups can be involved, working on the principles of collegiality and combining the efforts of specialists from various departments of the existing hierarchical structure. The formation of expert working groups in educational organizations is a common practice. Such groups can be more or less numerous and form hierarchies within themselves. But in a single system, their appearance is a sign of decentralization.

\section{Results and discussion}

Self-organization is accompanied by self-improvement, i.e. an increase in quality characteristics. This is ensured by the purposeful nature of self-organization while preserving its natural nature. Self-organization processes that take place against the background of interactions with the external environment remain autonomous [16]. Selfdeveloping systems seek positive (progressive) change. Such changes have the potential to accumulate and intensify, bringing the system into a new phase of development.

The system strengthens such influences through positive feedback, and the transition to a qualitatively different state is a positive feedback principle. Negative feedback stabilizes the system, does not lead to a new quality, but supports the functioning of the system - the principle of negative feedback. The two principles, despite the apparent contradiction reflected in their titles, were well suited for compromise. Moreover, the establishment of such a compromise is a condition for the development of self-organizing systems [17].

In examining the self-organization of systems as a phenomenon that exists objectively and requires substantive identification in the context of the study of the totality of 
organizational processes, it should be noted that autonomy is its most important characteristic. Following it, the system responds first and foremost to changes in internal structure and communication. It does so for certain internal purposes. The external environment acts as the initiator of change, but changes in the state of the system are achieved by changes in the internal state rather than by external determinants. The system uses a mechanism of cyclical self-reliance and self-development that is autonomous [18].

Self-organization, with its specific methodological prerequisites, creates the phenomenon of self-organization cycle. The notion of a self-organization cycle is commonly considered in relation to social systems [19]. A CPE with a social component (supplemented by an economic component) can be interpreted as a self-organizing cycle, with a series of blocks identified in the cycle and forward and backward linkages (Fig. 1). The base of the cycle shall be the following sequence of blocks.

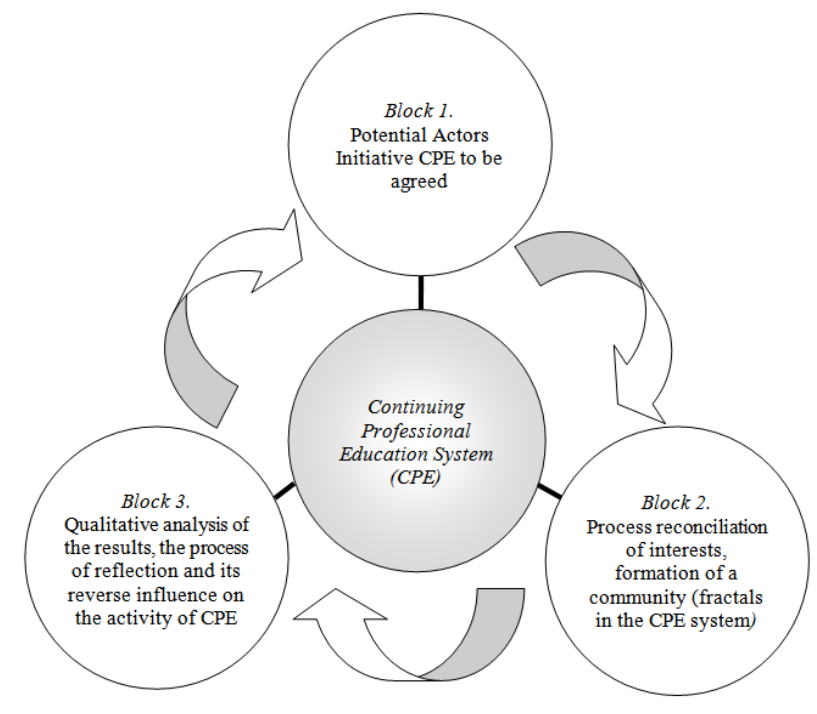

Fig. 1. Self-organizing cycle of continuing professional education.

The first block is the activation of subjects that are potentially capable of being included in the CPE system or in the fractals forming in it, increasing their initiative, understanding the logic of self-organization and striving for self-development based on the coordination of goals and interests.

The second block is the activation of the process of harmonization of interests, the formation of a community that is able to withstand the challenges of the external environment and effectively use the additional opportunities initiated by it.

The third block is the activation of the process of reflection, that is, cognition (analysis) of the results of coordinated actions, both at the level of the CPE system and each of its organizations. Reflection is accompanied by a qualitative analysis of the relationship between the result obtained and the goal declared in the process of harmonizing interests. In the course of such an analysis, deviations from the goal are revealed, and an appropriate adjustment is carried out, which makes it possible to continue actions to harmonize the interests of CPE subjects, to achieve their harmonization. The adjustment is the result of feedback action.

The self-organizing cycle, which carries all the signs of the mechanism of selforganization and self-development of the CPE system, is most active in the fractal structure of lifelong professional education, which reflects real opportunities for its so necessary in the modern period of innovative growth. Self-organization, in principle, can initiate new, 
but by no means necessary qualities. The reasons for this phenomenon lie in the plane of insufficient awareness of the subjects of the self-organization process (the CPE collective and its formal and informal divisions) and management and, accordingly, their insufficient understanding of strategically and tactically significant issues.

In such a situation, self-organization becomes not a stimulus for progress, but a stimulus for the development of destructive tendencies. The task of management in this case is being modified - it is necessary to strengthen the mechanism with elements of conscious organization, regulation, regulation and management in general, i.e. put certain barriers in the way of self-organization. It is important to keep in mind that such barriers remain in the field of rationality, provided they are prevented. With obvious manifestations of destructive tendencies, the barriers due to the property of self-organization and self-preservation are unlikely to be able to lead to the desired effect. It will become necessary to activate external influences opposing such self-organization, mobilize management tools from the leadership of organizations and the CPE system.

\section{Conclusions}

Evaluating lifelong professional education from the point of view of its susceptibility to the processes and tendencies of self-organization, one should speak of a high degree of susceptibility. Factors contributing to this susceptibility are:

- the nature of the activities of CPE organizations, which carries innovative, intellectual, creative components - categories that, in principle, cannot be formed outside of self-organization;

- belonging of the CPE system to social systems in which all the signs of selfdevelopment are observed and for which these signs are decisive;

- active human influence on the process of self-organization and the associated high uncertainty;

- a complex subject composition, both of individual organizations of CPE and of their entire system, which initiates the emergence of complex, multi-layered internal processes, self-organizing in various forms that are difficult to uniquely identify.

The last of the factors seems to be of fundamental importance. It is he who reflects the influence of a self-organizing and self-developing system of continuing professional education and, the results of its sustainable development and functioning on solving problems determined by the target guidelines of modern socio-economic policy - the large-scale implementation of innovations and related innovative management tools in the industry and economic spheres.

The stated provisions characterize the most important component of the theoretical and methodological basis of management of continuing professional education - its ability to self-development through the mechanism of selforganization. This mechanism acts as the main factor determining the stable functioning of the system of continuing professional education in periods of varying duration. Its organizational and economic nature allows the system of continuing professional education to adapt to the increasing dynamics of the external environment, achieving the given parameters of sustainability.

\section{References}


1. B. Mandelbrot, Fractal geometry of nature (Institute of Computer Research, Moscow, 2002)

2. H.-Y. Warneke, The Revolution in Entrepreneurial Culture. Fractal Enterprise (MAIK, Moscow, 1999)

3. O. Egorova et al, IOP Conf. Ser.: Mater. Sci. Eng., 698, 077029 (2019)

4. E. Rybnov et al, E3S Web of Conferences, 217, 11010 (2020)

5. T. Maleeva et al, IOP Conf. Ser.: Mater. Sci. Eng., 687, 044002 (2019)

6. N. Frolova, et al, E3S Web of Conf. 97, 06003 (2019)

7. E. Pesotskaya et al, IOP Conf. Ser.: Mater. Sci. Eng., 698, 077030 (2019)

8. I. A. Prigozhin, Organization: systems and people (Interprax, Moscow, 1983)

9. T. Yu. Ivanova, V. I. Prikhodko, Theory of Organization (St. Petersburg, 2004)

10. A. I. Kochetkova, Fundamentals of Management in conditions of chaos (Yurayt, Moscow, 2016)

11. Haken G. Information and Self-Organization: A Macroscopic Approach to Complex Systems (KomKniga, Moscow, 2005)

12. E. Trushkovskaya et al, IOP Conf. Ser.: Mater. Sci. Eng., 753, 032044 (2020) DOI:

13. R. Daft, Theory of Organization (Unity, Moscow, 2006)

14. E. Pesotskaya et al, IOP Conf. Ser.: Mater. Sci. Eng., 962022082 (2020)

15. L. G. Selyutina, Materials Science Forum, 931, 1113 (2018)

16. L. Egorova et al, IOP Conf. Ser.: Mater. Sci. Eng., 687, 044005 (2019)

17. T. V. Maleeva, L. G. Selyutina, Materials Science Forum, 931, 1118 (2018)

18. K. Bulgakova, L. Selyutina, Basics of investment projects selection for the implementation of regional investment programs in the sphere of social house building, Espacios, 39(26) (2018)

19. V. N. Kelasev, I. L. Pervova, Vestnik of Lobachevsky University of Nizhni Novgorod, 3 (35), 65 (2014) 